\title{
La actualidad del abordaje de la prostitución femenina en la prensa diaria española ${ }^{1}$
}

\author{
Andrea GUTIÉRREZ GARCÍA \\ Universidad Pontificia de Salamanca \\ agutierrezga@upsa.es
}

Recibido: 08/11/2012

Aceptado: 23/01/2013

\section{Resumen}

La prostitución es un tema complejo, donde se mezcla el patriarcado, la globalización capitalista, la pobreza generalizada, las diferencias entre países, la feminización de la pobreza, los movimientos migratorios...Por ello, para ser claramente comprendido, debe ser abordado en toda su extensión. Los medios de comunicación, como canales de información, tienen un importante papel en este sentido. El presente estudio pretende comprobar qué visión transmite la prensa española sobre este tema, para ello se centra en el análisis de las noticias sobre prostitución publicadas en la versión digital de los principales diarios, así como una revisión sobre los anuncios de contactos. Los resultados apuntan a que transmiten una imagen parcial, y con la publicación de los anuncios de contactos, legitiman la existencia de una institución que somete a las mujeres.

Palabras clave: prostitución, prensa, noticias, anuncios de contactos, patriarcado

\section{The Current Situation in the Tackling of Female Prostitution in the Spanish Daily Press}

\begin{abstract}
Prostitution is a complex issue, which involves the patriarchy, capitalist globalization, generalized poverty, the differences between countries, the feminization of poverty, migratory movements...Therefore, to be clearly understood, it must be tackled in all its extent. The mass media, as channels of information, have an important role in this sense. This study expects to confirm which prostitution view is transmitted by the Spanish Press. For that, we focus on the analysis of the news published by the main newspapers in its digital version, as well as a revision of the sex classified ads. The results point to a biased view of prostitution. Likewise, the publication of the sex ads legitimize the existence of an institution that submit women.
\end{abstract}

Keywords: Prostitution, Press, News, Sex Classified Ads, Patriarchy

\section{Referencia normalizada}

GUTIÉRREZ GARCÍA, Andrea (2013): "La actualidad del abordaje de la prostitución femenina en la prensa diaria española”. Estudios sobre el mensaje periodístico. Vol. 19. Núm. especial abril, págs.: 823831. Madrid, Servicio de Publicaciones de la Universidad Complutense.

Sumario: 1. La prostitución como institución que nos aleja de la igualdad. 2. Los medios de comunicación y su papel en el abordaje de la prostitución. 3. Metodología. 4. Resultados; 4.1. Posturas ante la prostitución; 4.2. Representación de las mujeres prostituidas; 4.3. Representación de los prostituidores; 4.4. Los anuncios de contactos. 5. Conclusiones. 6. Referencias bibliográficas.

${ }^{1}$ Este trabajo ha sido realizado gracias a la concesión de una beca predoctoral por la Consejería de Educación, Cultura y Turismo del Gobierno de La Rioja, asociada al III Plan Riojano de I+D+I 2008-2011. 


\section{La prostitución como institución que nos aleja de la igualdad}

En la actualidad, la demanda masculina de sexo resulta un negocio muy lucrativo que mantiene una tendencia creciente a nivel mundial (APRAMP, 2005), a pesar de que desde los años sesenta se han liberalizado las relaciones entre hombres y mujeres, y es relativamente fácil tener sexo sin necesidad de compromiso.

Para los hombres, acudir a la prostitución no es simplemente una forma de satisfacerse sexualmente, sino que es un espacio donde pueden ejercer su masculinidad tradicional, accediendo a una relación de poder. Mediante el pago obtienen el sometimiento de la mujer, convirtiéndola en una mercancía, un objeto de consumo a su disposición con el que no hace falta tener ninguna consideración. "Un hombre que viera en la mujer prostituida a un ser humano como él, señala Gimeno (2012:244), tendría dificultades para imponerle una actividad que a ella le está produciendo, como poco, displacer".

Para muchas mujeres, debido a las condiciones sociales actuales: precariedad económica, feminización de la pobreza, procesos migratorios, falta de alternativas vitales, consumismo generalizado... la prostitución es su única alternativa para sobrevivir, para otras es una condición impuesta por mafias o redes de trata que aprovechándose de su vulnerabilidad, las engañan y obligan a ejercer. Estas personas proceden de los países más pobres y desestructurados del mundo (de Miguel, 2012), si bien es cierto que actualmente, debido a la crisis, se han incorporado a la prostitución mujeres españolas.

La situación de alegalidad en la que se encuentra la prostitución en España, ha propiciado que coexistan con fuerza dos posturas ideológicas (Delgado y Gutiérrez, 2012), incluso dentro del mismo movimiento feminista. Por un lado, aquella que considera que ejercer la prostitución es un intercambio como el que se realiza en cualquier trabajo, y como tal debe ser regulado, gozando de derechos y deberes. Asimismo, señala el estigma como el gran problema de la prostitución, que desaparecería con la regulación, y cuya función es separar en dos a las mujeres, decentes (esposas y madres) y prostitutas, impidiendo la solidaridad entre ellas. La otra postura entiende que la prostitución es una forma de explotación y violencia, por cuanto es la misma mujer, y no otro servicio, lo que el prostituidor está interesado en comprar, con las implicaciones que esto tiene. Por tanto, desde esta postura se considera un atentado contra los derechos fundamentales y se reivindica su desaparición.

Lo cierto es que la regulación, en ninguno de los países donde se ha establecido, ha provocado un descenso en el número de mujeres tratadas y dedicadas a la prostitución, ni ha mejorado sus condiciones (Gimeno, 2012). Asimismo, su existencia nos afecta a todas y a todos (Díez, 2009), porque ratifica un modelo de sexualidad patriarcal y las relaciones de dominio de los varones sobre las mujeres.

\section{Los medios de comunicación y su papel en el abordaje de la prostitución}

La prostitución, como se ha visto, es un tema muy complejo, que debería ser abordado con todos sus matices por los medios de comunicación (Puñal, 2007). Éstos constituyen una de las principales fuentes de conocimiento e información, sobre las cuales, la sociedad construye una representación de ese problema. En la actualidad, los dos 
modos en que la prostitución está presente en la prensa son a través de la inclusión de los anuncios de contactos y en las noticias que se publican relacionadas con este tema.

En relación a los anuncios de contactos, España es actualmente el único país de la Unión Europea en el que la prensa generalista contiene anuncios de prostitución, a excepción de los diarios La Gaceta, La Razón, 20 minutos y Avui que tomaron la decisión de no publicarlos. La Comisión de Estudios del Consejo de Estado reunida en 2011 concluyó que estos anuncios atentan contra la dignidad de la mujer, implican un trato vejatorio y degradante y favorecen la transmisión de roles y estereotipos perpetuadores de la desigualdad. Asimismo, divulgan la idea de un género femenino sumiso, subordinado, servil e inferior y una imagen de las mujeres como mero objeto sexual.

En cuanto a las noticias sobre prostitución, las escasas investigaciones realizadas al respecto ponen de manifiesto la visión reduccionista y simplista que proporciona la prensa respecto a este fenómeno. Señalan, entre otras cosas, como los periódicos tienden a vincular mujer inmigrante que ejerce la prostitución con delincuencia y degradación (Brosa y Medina, 2012; Giró y Jarque, 2007), ponen de manifiesto como las noticias sobre prostitución son abordadas a menudo con cierto sensacionalismo y localizadas en algún distrito de la ciudad, lo que restringe las posibilidades de trascenderlo como asunto público que interesa a todas las personas. Asimismo, denuncian la ausencia de una perspectiva de género, social, política y económica al abordarlas, limitándose a la exposición de un hecho puntual (Puñal, 2007). De igual modo, se alerta sobre la utilización de un lenguaje sexista que mientras hace referencia a las mujeres como "prostitutas" o "putas", con todo el estigma que ello conlleva, se refiere a los hombres como clientes o consumidores, palabra neutra que designa al usuario de cualquier mercancía y que invisibiliza su contribución (Gutiérrez, 2012).

Para muchas personas, la prostitución es un tema que queda lejano de su experiencia personal directa, por lo que su conocimiento al respecto viene dado por la información que los periódicos publican (McCombs, 2006: 23). La representación que se construya en ellos, influirá en la actitud que mantenga la población al respecto. Por ello, es de suma importancia que los medios de comunicación trasladen el debate que se mantiene en las esferas asociativas, feministas y políticas sobre la legalización o la abolición de la prostitución y aporten información veraz y objetiva que permita a la ciudadanía construir una postura ideológica propia.

\section{Metodología}

El presente trabajo tiene como objetivo general comprobar qué visión transmite la prensa sobre el fenómeno de la prostitución. Para ello, nos centramos en el análisis de la representación que transmiten los diarios en sus noticias y a través de los anuncios de contactos, utilizando para ello una metodología cualitativa de análisis de contenido. Para la obtención de algunos datos estadísticos en relación a los anuncios de contactos se utilizó la metodología cuantitativa.

Por una parte, se analizaron las versiones digitales de los diarios de tirada nacional: Marca, As, El País, El Mundo y La Vanguardia. Se eligió la versión digital porque permitía buscar las noticias de manera más rápida, y se eligieron estos periódicos en base al último Estudio General de Medios (AIMC, 2011) que los señala como los 
más leídos. Las noticias fueron seleccionadas de las bases de datos de esos diarios y corresponden al mes de noviembre de 2011. La muestra de noticias la conformaron todas aquellas que contenían el término prostitución, en el titular o en el contenido.

Por otra parte, se recogieron los anuncios de contactos publicados en la edición impresa de los periódicos nacionales La Vanguardia, El País y $A B C$, éste último en la edición de Castilla y León, el periódico regional El Norte de Castilla y dos periódicos de edición local: El Adelanto de Salamanca y La Gaceta de Salamanca. El período de recogida de anuncios fue el mes de noviembre de 2011, sin embargo, para el análisis de datos sólo se utilizó la semana del 7 al 13 de noviembre de 2011 de manera arbitraria. Esta decisión se adoptó tras comprobar que los mismos anuncios se repetían a lo largo del tiempo, de manera que el análisis de los anuncios publicados durante una semana permitía obtener una muestra representativa de los mismos.

El material recogido en los medios de comunicación mencionados, ha sido analizado mediante la perspectiva de género.

\section{Resultados ${ }^{2}$}

\subsection{Posturas ante la prostitución}

Las noticias de prensa analizadas consideran el ejercicio de la prostitución como una profesión y a las mujeres prostituidas como trabajadoras del sexo. El lenguaje utilizado en los titulares hace referencia, en la mayor parte de los casos, a "prostitutas", pero en el cuerpo de la noticia el término se intercambia con el de "meretriz" o "trabajadoras del sexo": "Las trabajadoras del sexo subrayan en un comunicado que quieren espacios..." (El Mundo, 2/11/2011.)

Casi la totalidad de testimonios de mujeres prostituidas que se recogen, parten de la aceptación y normalización de la situación por parte de éstas y su lucha por mejorar sus condiciones, mediante la consecución de sus derechos como trabajadoras: “...es la actividad que hemos decidido escoger para trabajar","...ni víctimas, ni esclavas, nosotras decidimos" (El Mundo, 7/11/2011), "si en las calles sufrimos agresiones y robos, en el campo a oscuras y con una única entrada y salida corremos mayor peligro" "... pelear por nuestros derechos y llegaremos hasta donde haga falta por defenderlos" (El Mundo, 22/11/2011).

Cuando se habla de la prostitución como violencia de género, y por tanto se señala la necesidad de erradicarla, no se presentan testimonios en primera persona, sino que siempre son realizados por organizaciones, partidos políticos, etc. En definitiva, terceros que defienden esta postura: "La prostitución es una forma de violencia de género y las mujeres que la ejercen son víctimas a las que hay que facilitar y apoyar en

2 Para un análisis más en detalle se aconseja consultar GUTIÉRREZ, Andrea (2012): Análisis de la imagen de la mujer prostituida en las noticias de la prensa digital. Ponencia presentada en el I Congreso Internacional de Comunicación y Género (5-7 marzo), Sevi1la. GUTIÉRREZ, Andrea (2012): Prostitución y trata de seres humanos con fines de explotación sexual: análisis de la situación actual a través de los anuncios de contactos. Ponencia presentada en IV Congreso Universitario Nacional Investigación y Género (2122 junio), Sevilla. 
su proceso de recuperación personal y no multar..." (La Vanguardia, 5/11/2011), "el Plan prima la atención a las víctimas y se sitúa en la perspectiva de la defensa de los derechos humanos" (La Vanguardia, 5/11/2011).

En relación al delito de trata de personas con fines de explotación sexual, aparece de manera repetida nombrado erróneamente como tráfico de personas o simple explotación sexual. Asimismo, en estas noticias no se aporta información alguna sobre el delito, en qué consiste, los derechos y recursos que poseen las víctimas, etc. Tampoco se proporciona información sobre la situación en que se encuentran las mujeres. Sí que se incluyen las penas (mínimas) que recaen sobre los proxenetas o tratantes incautados, a menudo miembros de la policía o cargos políticos, y su impunidad fáctica: "Petición del propio Martínez Cardoso de apartarse temporalmente de las funciones de su cargo como responsable de vía pública hasta que se aclaren los hechos por los que ha sido imputado. No obstante, Martínez Cardoso seguirá manteniendo el acta de concejal y cuenta con el apoyo del gobierno municipal" (El País, 4/11/2011).

En cuanto a la prostitución infantil, apenas está presente en las noticias analizadas. En los artículos en los que se menciona, aparece una clara sanción al prostituidor por el abuso cometido. Todas las noticias se contextualizan fuera de España: "Los acusados de estos casos son lo peor de lo peor, obligando a niños a tener sexo por dinero...Las víctimas de estos crímenes son muy jóvenes, vulnerables y blanco fácil de los criminales" (El Mundo, 22/11/2011).

\subsection{Representación de las mujeres prostituidas}

A la hora de referirse a las mujeres prostituidas en las noticias, se utilizan los términos "putas", "prostitutas" o "meretrices":"Las putas eran suministradas por la pandilla del Carlton... y el bueno de DSK presuntamente, se beneficiaba a las damas" ( $E l$ Mundo, 17/11/2011). También aparecen denominadas como "material": "DSK: “¿Vienes a una discoteca pícara en Madrid conmigo (y con material)?"(Titular El Mundo, 10/11/2011, haciéndose eco de unas declaraciones de Strauss-Khan) o como "personas descarriadas": “...Y habrá paz para las descarriadas"(Titular El Mundo, 28/11/2011)

Se hace referencia a ellas como una categoría especial de mujeres, en oposición a las mujeres "decentes", fundamentalmente esposas y madres: "Se acabaron las jovencitas disfrazadas de grandes señoras con maquillaje excesivo y gigantes bolsos de Louis Vuitton. Ya no habría más actrices y bailarinas llegadas al mundo de la política como si de un concurso de belleza se tratara. Se cancelaban los vuelos en aviones del Gobierno usados como taxis para llevar prostitutas a la mansión que Silvio Berlusconi posee en Cerdeña. Elsa Antonioli es una mujer normal... Y cuando se sumerge en el trabajo y no le da tiempo a hacer la compra y la nevera se queda vacía, se encuentra con un marido muy tolerante... Tras años de desconcierto vuelve la normalidad a escena y se entierra la vulgaridad " (El País, 20/11/2011).

Se las define por la actividad que ejercen, reduciendo su identidad a ser prostituta: "Detienen a un hombre en Reus por atropellar a dos mujeres y darse a la fuga" (Titular El Mundo, 12/11/2011) vs. "Detenido por atropellar a dos prostitutas con su vehículo en Salou” (Titular La Vanguardia, 12/11/2011). 
Se las vincula asimismo con la delincuencia y las categorías peor consideradas de la sociedad: “... pins que mostraban a personajes de la Barcelona menos de escaparate como un latero, un vendedor ambulante de flores, un carterista, una prostituta y mossos pegando...El Ayuntamiento puede censurar cualquier objeto que crea que no está de acuerdo con la imagen de la ciudad. (El País, 3/11/2011).

Un gran número de noticias recogen las agresiones que sufren las mujeres prostituidas, a manos de sus clientes. Sin embargo, en todas ellas se aborda la noticia como un suceso puntual, ninguna lo vincula a la violencia basada en el género, incluyendo en ocasiones elementos de corte sensacionalista: "Condenan a 14 años de cárcel al hombre que mató y descuartizó a una prostituta" (Titular El Mundo, 4/11/2011), "Según han informado fuentes policiales, la víctima y agresor mantenían contactos sexuales esporádicos, pero no tenían una relación estable de pareja. Por tanto, los agentes descartan que se trate de un caso de violencia de género y se inclinan por un enfrentamiento puntual" (La Vanguardia, 4/11/2011), “...ha llegado a quedarse en bragas ante el tribunal para mostrar las heridas que le causó...., explicó cómo acordaron el precio - 60 euros por media hora- y después el acusado, al que le piden 10 años por asesinato en grado de tentativa, la agredió... ha llegado a bajarse los leotardos para mostrar al juez daños en sus inglés" (El Mundo, 28/11/2011).

\subsection{Representación de los prostituidores}

Se incluyen argumentos que tienden a justificar el comportamiento de los prostituidores, llamados clientes, que acuden a obtener relaciones sexuales a cambio de dinero: "Sus correrías nocturnas con la pandilla de Lille eran, para Dominique, una bocanada de aire que le sacaba de su rutina con los intelectuales...se sentía muy solo y aburrido en Washington" (El Mundo, 17/11/2011).

Se habla también de ella como una actividad masculina vinculada al ocio, al entretenimiento, o incluso se presenta como una nueva manera de cerrar negocios o como premio ante la finalización exitosa de un trabajo. Las noticias, relacionadas con prostitución de lujo, están protagonizadas por personas relevantes del mundo de la política, empresarios o futbolistas. “... proclamar que la victoria del $20 \mathrm{~N}$ habría que celebrarla con 'vino y mujeres"” (El Mundo, 21/11/2011). En la mayoría de ellas, el escándalo que despierta la noticia es debido al pago de esos servicios con fondos públicos: "Emarsa era una sociedad pública, encargada de la depuración del agua de Valencia, liquidada el año pasado por el PP...Y ahí estaban esos directivos para utilizar -o permitir- que los fondos de la empresa... sufragara el pago de circuitos de spa para sus directivos o el pago de noches de hotel que incluía la retribución a prostitutas" (El País, 26/11/2011).

\subsection{Los anuncios de contactos}

En la semana del 7 al 13 de noviembre de 2011 aparecen 3271 anuncios en los periódicos consultados, lo que suponen unas ganancias de 57801,38 euros semanales.

En cuanto al género de quien se oferta, se observa que en el $86,12 \%$ de los anuncios hay alguna referencia explícita al mismo o se puede deducir del contenido. De éstos, en el $83,64 \%$ son las mujeres las protagonistas frente al $4,8 \%$ de hombres que 
ofrecen sus servicios. En el 8,45\% de los casos son travestis. El resto de anuncios son escritos por una tercera persona o agencia que precisa reclutar mujeres u hombres para ofrecer servicios sexuales; y en el menor número de ocasiones son parejas. Asimismo, mientras que en la totalidad de anuncios de mujeres éstos van dirigidos a público masculino, en los hombres encontramos un gran porcentaje de anuncios homosexuales.

La nacionalidad aparece reflejada en un $34,34 \%$ de los anuncios siendo, como grupo, mayoritarias las españolas. Sin embargo aparecen 23 nacionalidades distintas que representan el 55,6\% del total. En cuanto a la edad, el 61,71\% tienen entre 18-30 años, el resto se distribuyen hasta los 65 años.

En relación a la práctica sexual ofertada sólo aparece de manera explícita en el $42,71 \%$ de los anuncios, siendo los masajes y “todos los servicio, sin tabúes” los más numerosos.

Las mujeres se describen como "guarronas", "niñatas", "morbosas", "viciosas", "supercomplacientes", "discretas"... con el objetivo de atraer la atención de posibles clientes.

Se observa como determinados anuncios están escritos en tercera persona. También aparecen anuncios de establecimientos donde se ofertan los servicios sexuales de varias mujeres durante las 24 horas del día: "tenemos lo que buscas rubia, ojos verdes, 24 años, mulata, guapa, travesty, todos los servicios, 24 horas"(La Gaceta, 910/11/2011).

Además, son muy frecuentes las alusiones a la novedad o la renovación de las mujeres: "Lolitas, lolitas, renovación, 8 Jovencitas te harán descubrir tus fantasías. Sí a todo. L.climat. Hab.bañera..." (La Vanguardia, 7/11/2011).

\section{Conclusiones}

Lo primero que destaca en el análisis de la cobertura mediática de la prostitución, es la escasez de informaciones que hagan referencia a la misma, en contraste con el amplio espacio ocupado por los anuncios de relax, esto es explicado por Fagoaga (2007: 201) como resultado del carácter patriarcal de los medios de comunicación, la sumisión a una agenda marcada desde fuera y la falta de recursos para informar e investigar. Asimismo, la información aportada se reduce a noticias en momentos puntuales, generalmente recogidas en la sección de sucesos, y presenta una visión simplista de la prostitución, en ocasiones incluso justificada. Los testimonios en primera persona que se recogen, son los de aquellas mujeres que declaran haber elegido voluntariamente ejercer la prostitución, transmitiendo la idea de libre elección. Esta carencia de pluralidad y reflexión puede confundir a la población y originar ideas sesgadas, orientando su actitud de manera favorable a la prostitución y a su regulación, siempre que no llegue a ocasionarles conflictos vecinales.

Por otra parte, el acercamiento a los anuncios de contactos permite darse cuenta de que la prostitución es una cuestión de género, porque son mujeres la que la ejercen y hombres las que la usan. Asimismo, se pone de manifiesto la falacia que asocia prostitución y sexo liberador, pues ésta no hace otra cosa que mantener y reafirmar las construcciones sociosexuales más tradicionales y opresivas, en la que ellos practican sexo por placer y ellas ponen su cuerpo a disposición del placer de ellos: "sí a todo", 
"dispuestas a todo", "disponibles 24 horas". Además, las mujeres aparecen como productos de consumo variados, renovados y accesibles para cualquier hombre, cuya atracción no radica fundamentalmente en la práctica que ofrecen, como ocurre en cualquier otra actividad.

Por todo ello, fruto del análisis realizado, se sugiere a los medios de comunicación que vayan más allá en sus informaciones, que ahonden en las causas, consecuencias y el contexto actual de la prostitución, que huyan del sensacionalismo, la frivolidad y el morbo en la elaboración de éstas, que les den voz a las mujeres que ejercen o han ejercido la prostitución, pero a todas, y que fomenten el debate para que llegue al público en general. Asimismo, deberían renunciar a la publicación de los anuncios de contactos que transmiten roles y estereotipos de las mujeres, normalizan la existencia de prostitución, y nos alejan de la consecución de la igualdad.

\section{Referencias bibliográficas}

AIMC (2011): "Estudio General de Medios 2011", en: http://www.aimc.es//-DatosEGM-Resumen-General-.html [fecha de consulta: 1 de enero de 2012]

APRAMP (2005): La prostitución, claves básicas para reflexionar sobre un problema. Madrid, Fundación Mujeres.

BROSA HERNÁNDEZ, Julia y MEDINA BRAVO, Pilar (2012): "Representación de la prostitución inmigrante en la prensa. El caso de las prostitutas del barrio del Raval de Barcelona”. Estudios sobre el mensaje periodístico. Vol. 18, núm. 1, págs.: 259-273. Madrid, Servicio de Publicaciones de la Universidad Complutense.

COMISIÓN DE ESTUDIOS DEL CONSEJO DE ESTADO (2011): "Estado de situación y posibles actuaciones contra anuncios de contenido sexual y prostitución", en : http://www.consejo-estado.es/pdf/ [fecha de consulta: 9 de enero de 2012]

DE MIGUEL, Ana (2012): "La prostitución de mujeres, una escuela de desigualdad humana". Revista Europea de Derechos Fundamentales, 19, pp.49-74.

DELGADO, Carmen y GUTIÉRREZ, Andrea (2012, en prensa): "Prostitución: notas para un análisis psicosocial. De la coacción al consentimiento", en Igualdad:retos para el S XXI. Santiago de Compostela, Andavira.

DÍEZ, Enrique Javier (2009): "Prostitución y violencia de género", en Nómadas, revista crítica de ciencias sociales y jurídicas, 24, http://www.ucm.es/info/nomadas/24/enriquediez.pdf. [fecha de consulta: 12 de octubre de 2011]

FAGOAGA, Concha (2006): "Los discursos de prostitución en la prensa española", en Congreso Internacional de Derechos Humanos y prostitución. Madrid, Dirección General de Igualdad de Oportunidades y Ayuntamiento de Madrid, pp. 200211.

GIMENO, Beatriz (2012): La prostitución . Barcelona, Bellaterra.

GIRÓ, Xavier y JARQUE, José Manuel (2007): “El discurso editorial sobre inmigración de El País, La Vanguardia, El periódico y Avui, desde El Ejido hasta la cumbre europea de Sevilla (1999-2002)", en ZAPATA, Ricard y VAN DIJK, Teun 
A.Van: Discursos sobre la inmigración en España. Barcelona, Bellaterra, pp.1744.

GUTIÉRREZ, Andrea (2012) “ Análisis de la imagen de la mujer prostituida en las noticias de la prensa digital", en I Congreso Internacional de Comunicación y Género. Sevilla, Facultad de Comunicación, pp. 846-871.

MC COMBS, Maxwell (2006): Estableciendo la agenda: el impacto de los medios en la opinión pública y el conocimiento. Barcelona, Paidós.

PUÑAL, Ana Belén (2007): "El tratamiento de la prostitución en los medios. Algunas recomendaciones", en MENÉNDEZ, $\mathrm{M}^{\mathrm{a}}$ Isabel (Ed.): Buenas prácticas periodísticas desde la perspectiva de género. Madrid, AMECO, pp. 145-161.

\section{Andrea GUTIÉRREZ GARCÍA}

Universidad Pontificia de Salamanca

Personal investigador en formación agutierrezga@upsa.es 